\title{
Deux modèles de causalité, deux théories de la liberté: À propos de deux interprétations de la proposition I du Livre des causes
}

\author{
Olivier Boulnois \\ École Pratique des Hautes Études, Paris
}

Quelle place le Livre des causes occupe-t-il dans l' histoire de la métaphysique? Un de ses apports les plus décisifs est certainement l'invention des concepts de «cause première» et de «cause seconde». Je m'intéresserai ici à la manière dont la pensée de la liberté s'est articulée à ce dédoublement du concept de cause, dans l'histoire de la réception de la première proposition du Livre des causes. En effet, originellement, bien avant de s' opposer dans la troisième antinomie kantienne ${ }^{1}$, liberté et causalité constituaient les deux faces d' une même médaille: l'insertion du libre arbitre dans le monde fait appel au concept de responsabilité, c' est-à-dire de causalité (je ne dois répondre d' un acte que dans la mesure où j'en suis le principe et la cause), et réciproquement, la causalité indique en quoi un être a pris part à une action².

En quoi ces deux concepts, propres au Livre des causes, permettent-ils de penser le libre arbitre, et d'articuler la liberté des agents avec l'ordre du monde? Je me concentrerai sur la place occupée par la première proposition du Livre des causes au sein du questionnaire élaboré par la philosophie médiévale, sur la relation entre le libre arbitre et la causalité. Nous devons nous demander: le libre arbitre est-il une cause seconde parmi d' autres ou fait-il exception au statut général des causes secondes? Pour répondre à cette question directrice, comme nous le verrons, les textes les plus éclairants ne sont pas seulement les commentaires sur le Liber, mais aussi des traités philosophiques ou théologiques autonomes.

Notre question directrice prend donc une double forme: 1. qu' est-ce que le concept de cause première? Et corrélativement: qu' est-ce que le concept de cause seconde? (I) Puis 2. Comment ces deux concepts s'articulent-ils? Nous verrons qu'il y a deux modèles dominants dans la manière de les articuler: la

1 Kant, Kritik der reinen Vernunft, Ak. III, 308-311 (éd. Delamarre, Marty 1980, I, 1102-1105).

2 Le terme grec aitia (cause), d' où vient aitios (responsable), vient de la racine *aiteô, «avoir part, réclamer sa part, demander» (Chantraine 1968, p. 41a). 
cause primordiale peut soit précontenir la cause seconde, lui conférer l'être et l' agir (II), soit s' ajouter à la cause seconde en lui donnant l'être mais non l' agir (III et IV).

\section{Les concepts de cause première et cause seconde}

Que signifient, et d' où viennent ces deux concepts? Dans ses Écrits sur la grâce, Pascal, afin de penser la relation entre le libre arbitre humain et la volonté divine, estime nécessaire d'employer les concepts de «première cause», et de «cause seconde»:

Encore qu' on puisse attribuer les actions ou à la volonté de l'homme ou à la volonté de Dieu, et qu'en cela ces deux causes semblent y concourir également, néanmoins il y a cette entière différence, qu' on peut attribuer l'action à la seule volonté de Dieu, à l'exclusion de la volonté de l'homme; au lieu qu'elle ne peut jamais être attribuée à la seule volonté de l'homme à l' exclusion de celle de Dieu. Car quand on dit que l'action vient de notre volonté, on considère la volonté humaine comme cause seconde, mais non pas comme première cause; mais quand on cherche la première cause, on l'attribue à la seule volonté de Dieu, et on en exclut la volonté de l'homme ${ }^{3}$.

Les concepts de cause première et cause seconde appartiennent à une histoire de longue durée (huit siècles séparent le Liber de causis des réflexions de Pascal). Chez Pascal, ils instrumentent deux thèses: 1. L'action humaine résulte du «concours» de deux causes, la volonté de Dieu et la volonté de l'homme. 2. Elles s'articulent selon un ordre, comme la «première» à la «seconde». Une conclusion en découle: on peut éventuellement «attribuer l'action» à la seule volonté de Dieu, mais non à la seule volonté de l'homme; la cause divine est prédominante; c'est une théorie de l' «attribution», c' est-à-dire de l'imputation de la responsabilité principale (à Dieu $)^{4}$.

3 Pascal, Écrits sur la grâce, Écrit XII, CEuvres complètes II, (éd. Le Guern 2000, p. 294; cf. Écrit I, p. 219: «nous trouverons [...] que Dieu opère sans que l'homme coopère, et que l'homme coopère avec Dieu»); voir Augustin, Contre deux Épitres de Pélage II, 9, 20-21: «Dans un cas Dieu coopère avec l'homme qui agit, tandis que dans l' autre c' est lui seul qui agit. Dieu fait en l'homme beaucoup de bien que l'homme ne fait pas; mais l'homme ne fait aucun bien que Dieu ne fasse faire à l' homme» (BA 23, 452).

4 Cette théorie de l'attribution a été étudiée par A. de Libera (Libera 2007, p. 98-99). 
Ces deux thèses (et leur conclusion) supposent un raisonnement serré:

1. L'action est un attribut de la volonté, qui en est la cause.

2. Les actions humaines sont des attributs de la volonté humaine.

3. Mais la volonté humaine dépend à son tour de la volonté divine, ou «première cause »; elle en estl' effet:l' homme veut ce que Dieu veut qu' il veuille; il fait donc ce que Dieu veut.

4. Par conséquent, on doit attribuer l' action au concours de ces deux causes, la volonté divine comme première cause, la volonté humaine comme cause seconde.

5. Pour la même raison, on peut attribuer l' action à la seule volonté de Dieu: la volonté de l'homme découlant nécessairement, déterminément, de la seule décision volontaire de Dieu.

6. Mais pour la même raison, on ne peut pas attribuer l'action à l' homme seul.

Mais d'où proviennent les concepts de cause première et de cause seconde? Clairement, le concept est emprunté à la scolastique, et pour Pascal, à saint Thomas ${ }^{5}$. Mais Thomas d'Aquin les attribue à son tour au Liber de causis: «dans les réalités naturelles, la cause première ( prima) influe davantage sur l' effet de la cause seconde que ne le fait aussi la cause seconde elle-même, comme il est dit dans le Livre des causes $»^{6}$. D'emblée, Thomas lie les concepts de cause seconde et de cause première par un principe paradoxal: la cause première, éloignée, est plus efficace que la cause seconde, pourtant prochaine.

Et pour l'ensemble de la scolastique, c'est bien du Livre des causes, que viennent les concepts de cause première, et de cause seconde. Ce traité définit leur relation en trois principes formant un paradoxe: 1. la cause première agit davantage que les causes secondes; 2 . elle peut produire leur effet sans elles; 3 . elle le produit avant elles.

L'incipit du Livre des causes prend la forme d'un principe premier absolu, d'un axiome non-déductible mais fondamental: «Toute cause primordiale (primaria) influe davantage sur son effet que la cause universelle seconde» (première proposition, que j' appelerai désormais le «principe $1 »)^{7}$. Même les effets de la cause seconde sont causés par la cause première, cause de celle-

5 Que Pascal étudiait de près lorsqu' il discutait de la scolastique jésuite; cf. de Franceschi 2018, p. 34-35, 104, 120-121, 286-288, 307-309, 315-318, 434-439.

6 Thomas d'Aquin, Scriptum super libros Sententiarum II, d. 44, q. 2, a. 3 expos.: in naturalibus causa prima plus influit supra causatum causae secundae quam etiam ipsa causa secunda, ut in Libro de causis dicitur.

7 Liber de causis I, §. 1: Omnis causa primaria plus est influens super causatum suum quam causa universalis secunda (Pattin 1966, p. 46); trad. Boulnois et al. 199o, p. 39 (je corrige toutes les traductions). 
ci. On peut ainsi dire que l'effet procède davantage de la cause divine que de sa cause prochaine, puisque l' efficace de la cause seconde lui vient de la cause première, et ne fait pas nombre avec celui-ci.

Dans le Liber de causis, deux propositions découlent du principe initial:

1. «Donc, lorsque la cause universelle seconde ôte sa puissance à une chose, la cause universelle première ne lui ôte pas la sienne» (commentaire que j' appelerai «principe $2 »)^{8}$. Le Liber ne parle pas ici de plusieurs causes secondes, mais de la cause seconde qui est la cause «universelle», la cause de toutes choses. Or selon lui, la cause première agit toujours, même si la cause seconde est suspendue. Elle n'est pas la seule créatrice de l' acte de cette cause seconde (par exemple, l'intellect); mais elle donne à celle-ci la puissance de causer et même l' efficace causale (l'influx) par lequel elle exerce sa causalité. Puisque la cause seconde n' agit pas sans elle, nous n'avons pas affaire à deux causes partielles qui s' additionneraient pour produire un effet commun. La cause première agit à travers l'action de la cause seconde et elle ne fait pas nombre avec elle, mais lorsque celleci cesse d'agir, elle continue de le faire: elle peut donc conserver dans l'être une chose même si la cause seconde n'existe plus ou n'est plus active.

2. Le commentaire se prolonge en un autre principe, le «principe 3 »: «La cause universelle première agit sur l' effet de la cause seconde avant que n'agisse sur lui la cause universelle seconde qui l' accompagne ${ }^{9}$. Puisqu' une cause produit son dérivé, elle agit avant le dérivé pour le produire et produire son effet. Dans l'ordre de la causalité, elle est antérieure; elle est donc plus fondamentale que toute autre cause.

Ce couple de concepts peut-il avoir une autre source? - Certes, l'idée d'une chaîne de causes, qui implique une cause première et une série de causes et d' effets successifs, n' est pas propre au Livre des causes. On trouve par exemple dans le De potestate de saint Anselme: «Toute cause a des causes, [et ce] jusqu'à la cause suprême de tout, Dieu, qui, parce qu'il est la cause de tout ce qui est quelque chose, n'a aucune cause ${ }^{10}$. Anselme admet une série de causes,

8 Liber de causis I, §. 2: Cum ergo removet causa universalis secunda virtutem suam a re, causa universalis prima non aufert virtutem suam ab ea (Pattin 1966, p. 46; Boulnois et al 199o, p. 39).

$9 \quad$ Liber de causis I, §. 3: Quod est quia causa universalis prima agit in causatum causae secundae, antequam agat in ipsum causa universalis secunda quae sequitur ipsum (Pattin 1966, p. 46 ; Boulnois et al 1990. p. 39).

10 Anselme, De potestate, Fragmenta philosophica II, p. 339; trad. Galonnier, in Corbin 199o, p. 424: Habet enim omnis causa causas usque ad supremam omnium causam deum, qui cum sit causa omnium quae aliquid sunt, nullam habet causam. 
et il pose au principe de cette série une cause de toutes choses, Dieu. Mais il n' appelle pas cette cause «première ». Et surtout, il ne lui donne pas la capacité d' envelopper la série des causes finies et d' agir au plus intime d' elles-mêmes, voire avant celles-ci. Car ce qui caractérise le concept des causes secondes, c'est précisément l'idée que la cause première les précède et précède même leur propre action: qu' elle agit davantage qu' elles, sans elles, et avant elles.

De même, Gundissalinus distingue entre une cause primordiale et une cause secondaire: «Au commencement, il y avait une double cause: à savoir une cause primordiale (primaria) et une cause secondaire (secundaria). La cause primordiale est Dieu; la cause secondaire est son instrument (instrumentum), et d' elle viennent les mêmes œuvres ${ }^{11}$. On trouve bien ici l'idée d' un concours hiérarchique entre les causes, mais précisément, il manque encore à cette articulation des causes le lien paradoxal par lequel la cause première agit davantage que la cause seconde (le principe 1). Ici, la cause «secondaire» est considérée comme l' «instrument» par lequel Dieu arrive à son œuvre, mais rien n'indique que Dieu puisse produire l'effet de cet instrument avant l'instrument, ou sans lui.

Or la thèse de Pascal est une application particulière à la volonté du principe 2 ; et celui-ci découle du principe 1. Par conséquent, la première proposition du Livre des causes structure tout un champ conceptuel: elle permet de penser à la fois l' articulation des causes premières et des causes secondes, la conservation de l'être par la cause première, et (en particulier) le concours évoqué par Pascal entre volonté divine et liberté humaine, entre grâce et libre arbitre.

Les concepts de cause première et de cause seconde nouent ensemble une matrice de problèmes. Ils permettent de penser le rapport entre Dieu et les créatures, mais cette difficulté s' exprime à son tour en un complexe de questions et de réponses. Le concours entre la cause première et la cause seconde permet de penser la transcendance de la puissance du premier en même temps que l' autonomie des créatures, d' affirmer à la fois que les causes n' agissent que dans la dépendance du Principe, et que celui-ci possède une causalité plus radicale que la leur. La puissance divine précède et rend possible toute la causalité

11 Gundissalinus, Von dem Hervorgange der Welt (De processione mundi), p. 51: Bipertita fuit in principio causa: scilicet primaria et secundaria. Primaria causa Deus est; secundaria instrumentum eius de ipsis eiusdem operibus. Sed primae secundis praelatae sunt auctoritatis. La différence de vocabulaire ne suffit pas à parler de concepts distincts: les commentateurs du Livre des causes emploient aussi l' expression causa primaria et causa secundaria pour parler des causes première et seconde du Liber (par exemple Siger de Brabant, Quaestiones super Librum de causis, q. 25, ed. Marlasca 1972, q. 2, p. 40: non oportet quod causa primaria possit effectum producere sine causa secundaria); mais le $\S .1$ du Liber de causis emploie déjà l' expression causa primaria. 
des créatures, mais elle s' exerce à travers elles. Cela nous conduit à la première difficulté: si la première cause (Dieu) agit davantage que la cause universelle seconde (1) sans elle (2), et avant elle (3), cela veut-il dire que la cause s' exprime à travers les causes secondes, et qu' elle peut causer l' effet des causes secondes sans elles? ou bien seulement qu' elle peut causer autrement la cause universelle, et indirectement ses effets? En un mot, peut-elle ou non court-circuiter la cause seconde pour produire directement ses effets? C'est la question de la liberté et de la toute-puissance du Principe qui est ici engagée.

Analysons d' abord ce que dit notre proposition:

1. La causalité revient davantage à la cause première qu'à la cause universelle seconde. La cause première est davantage cause que son effet, la cause seconde (principe 1, §.1).

2. Réciproquement, la cause seconde étant l'effet de la cause première, elle en dépend pour agir et elle a moins de puissance qu' elle.

3. Les effets des causes secondes résultent d'un concours de causalité entre l'action de la cause première et celle de la cause seconde.

4. La cause première continue de causer une chose même lorsque la cause seconde n'y concourt plus: elle la conserve ( $\$ .2$, principe 2 ).

5. La cause première est antérieure à la cause seconde ( $\$ .3$, principe 3$)$.

Mais quelle est l' origine de cette articulation? Pouvons-nous atteindre un socle généalogique plus ancien que le Liber? - Dans la théologie de Proclus, le bien, l'intellect et l'âme forment une série hiérarchique. Le bien est à la fois la cause la plus universelle et le principe le plus intime de toute causalité. Mais de ce fait, il agit avant, avec et après son effet, l'intellect, que le Livre des causes appellera cause seconde. Il en découle que tout ce dontl'intellect est cause, le bien en est cause, mais que la réciproque n' est pas vraie:l'intellect n' est pas cause aussi de tout ce que produit le bien (à commencer par sa propre causalité); de même, tout ce dont l'âme est cause, l'intellect aussi en est cause, mais l' inverse n' est pas vrai. L'antériorité implique une dissymétrie, une non-réciprocité.

Cette thèse est empruntée à Proclus: le principe qui donne à la cause seconde sa puissance de produire possède cette puissance à titre primordial et suréminent: «Toute cause à la fois agit avant son effet et fait subsister après lui un plus grand nombre $\langle$ de subsistants $\rangle »^{12}$. Mais ici, Proclus parle universellement de «toute cause» (pan aition, il s' agit de toute cause première dans une série causale), et de surcroît cette cause est «hypostatique » (à travers son effet, elle fait subsister plusieurs êtres). Tandis que dans le Livre des causes, le

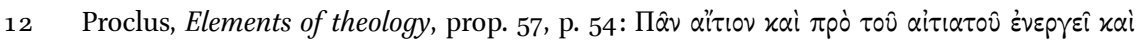

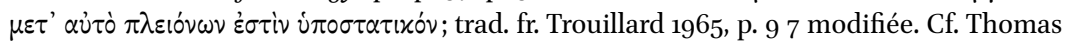
d'Aquin, In Librum de causis expositio, §10, éd. Pera, p. 5. 
principe est considéré comme unique; il engendre un unique effet, que le Livre appelle la «cause seconde universelle» (§ 1). Selon Proclus, toute cause est plus parfaite et plus puissante que ses subordonnés. Ainsi, elle peut être cause d'un plus grand nombre d'effets. Puisqu' elle produit son dérivé, elle agit avant le dérivé pour le produire. Elle agit avant son effet, ce qui correspond au principe (3). Mais aussi après lui, puisqu' elle forme et fait subsister après lui le reste des êtres. Agissant avant lui et après lui, elle peut agir sans lui, ce qui correspond au principe (2).

Dès lors, la hiérarchie entre cause première et cause seconde s' exprime dans une relation non-réciproque. La puissance du premier est inamissible. La cause première agit toujours, même si la cause seconde est suspendue. La cause première n' est pas la seule créatrice de l'acte de la cause seconde (par exemple, l'âme): elle donne à la cause seconde la puissance de causer et même l' efficace causale (l'influx) par lequel celle-ci exerce sa causalité. La cause première agit à travers l'action de la cause seconde, mais elle ne fait pas nombre avec elle. Il ne s' agit pas de deux causes partielles concourantes qui s' additionneraient pour produire un effet commun.

La puissance divine précède et rend possible toute la causalité des créatures, mais elle s' exerce à travers elles. Les créatures ne peuvent rien sans Dieu, mais Dieu n' agit pas sans elles. Cela pose des problèmes métaphysiques et théologiques redoutables: chez Proclus et dans le Livre des causes, la production du monde se fait selon une cascade d'émanations: le Principe cause l'intellect, qui produit l'âme, qui produit la nature, etc. La création des êtres sensibles est seulement médiate, et la Providence est limitée par l'ordre de la nature. Mais cette thèse est-elle compatible avec une théologie révélée admettant un Dieu libre et tout-puissant? C'est le problème que devront aborder les lecteurs et commentateurs médiévaux du Liber de causis.

Je distinguerai deux grandes interprétations médiévales du Liber de causis, en me limitant principalement à Thomas d'Aquin, Duns Scot et Guillaume d'Ockham, mais ces trois auteurs ne représentent qu'une coupe sagittale dans un champ beaucoup plus complexe, où s'entrecroisent les concepts, les problèmes et les textes, et qui mériterait d'être explorés plus précisément.

Le premier modèle d'interprétation du Liber de causis: Thomas d'Aquin

Dans son commentaire du Liber, Thomas désamorce la dimension néoplatonicienne de l' opuscule. Il n' ignore pas que les Platonici soutenaient que les intellects séparés procèdent par participation de formes séparées, «qu'ils appe- 
laient dieux $»^{13}$. Mais sur la relation de l' intellect aux intelligibles, il faut suivre la position d'Aristote, qui sur ce point concorde davantage avec la foi chrétienne (est magis consona fidei Christianae): «nous ne posons pas de formes séparées supérieures à l' ordre des intellects», mais seulement un «bien séparé», un bien «extérieur» (extrinsecum) vers lequel tend tout l'univers. Il faut donc dire que les intellects «suivent» (consequuntur) ces formes intelligibles, mais «par participation à la première forme séparée, qui est le bien pur, à savoir Dieu $»^{14}$.

De même, on n' acceptera pas l' idée que la cause seconde reçoive de la première la faculté de créer d' autres êtres. Il faut réserver à Dieu cette prérogative $^{15}$. Dans l'Écrit sur les Sentences, Thomas associe cette doctrine à la pensée d'Avicenne, selon le principe ex uno non fit nisi unum ${ }^{16}$. Et dans son commentaire de la proposition III du Liber, il soutient qu' une telle doctrine n'est pas conforme à l'intention du livre, puisque selon le Liber, la cause première communique l'être à tous les étants, tandis que l' intellect ne fait que communiquer la pensée à tous les intelligents ${ }^{17}$. Dès lors, on ne peut plus lire le Liber de causis

13 Thomas d' Aquin, Super Librum de causis expositio, Lectio 3 (éd. Saffrey 20oo, p. 18): omnes huiusmodi formas sic subsistentes 'deos' vocabant.

14 Thomas d'Aquin, Super Librum de causis expositio, Lectio 10 (éd. Saffrey 1954, p. 67-68): Sed quia, secundum sententiam Aristotelis quae circa hoc est magis consona fidei Christianae, non ponimus alias formas separatas supra intellectuum ordinem, sed ipsum bonum separatum ad quod totum universum ordinatur sicut ad bonum extrinsecum, ut dicitur in XII Metaphysicae, oportet nos dicere quod, sicut Platonici dicebant intellectus separatos ex participatione diversarum formarum separatarum diversas intelligibiles species consequi, ita nos dicamus quod consequuntur huiusmodi intelligibiles species ex participatione primae formae separatae, quae est bonitas pura, scilicet Dei. A. de Libera a souligné cette christianisation du Liber de causis par Thomas (Libera 1990, p. 347-378, notamment p. 370-371).

15 Thomas d'Aquin, Super Librum de causis expositio, Lectio 3 (éd. Saffrey 20oo, p. 23). Comme dit H.-D. Saffrey dans son «Introduction» (parlant du point de vue thomiste): «Non moins fondamentale assurément est la transpostion du système néoplatonicien des «processions » en celui d'une véritable création. Encore [qu'] il semble bien que l' auteur du Liber soit resté à mi-chemin. Car il y a bien une création au sens fort de la cause première à la prima rerum creata, qui est l'esse, mais cet esse est l'esse superius, c' est-àdire une espèce de primum esse, qui sera cause de cette perfection dans les autres êtres» (p. XXXI).

16 Thomas d'Aquin, Scriptum super libros Sententiarum II, d. 18, q. 2, a. 2: Posuerunt enim a primo Principio quod Deus est, eo quod est unum et simplex, non potuisse immediate plura prodire, [...] et ideo a Primo immediate procedit Intelligentia prima, [...] et ita ab hac Intelligentia $[. .$.$] dicunt effluere secundam Intelligentiam.$

17 Thomas d'Aquin, Super Librum de causis expositio, Lectio 3 (éd. Saffrey 200o, p. 23): Esse enim quod est communissimum, diffunditur in omnia a causa prima. Sed intelligere non communicatur omnibus ab intelligentia, sed quibusdam, praesupponendo esse quod habent a primo. 
comme témoignage d'une hiérarchie d'hypostases, mais bien comme pensée de la création réservée au premier Principe. La cause première ne peut plus être que l' unique Créateur confessé par la foi chrétienne ${ }^{18}$.

Dans son Écrit sur les Sentences, saint Thomas d'Aquin présente l' articulation des causes secondes comme un juste milieu entre deux extrêmes, celui du kalâm (de la dialectique théologique arabe), qui nie toute causalité des causes secondes, celui des philosophes, qui transmettent au contraire la puissance créatrice aux causes secondes.

\subsection{Contre la négation des causes secondes (le kalâm)}

Le premier extrême est l'occasionnalisme du kalâm (l'apologétique musulmane). Selon celui-ci, «Dieu opère immédiatement toutes choses, de sorte que rien d'autre n'est cause de rien; ce n' est pas le feu qui chauffe, mais Dieu; ni la main qui est mue $\langle$ par nous $\rangle$, mais Dieu qui cause son mouvement, etc. ${ }^{19}$. Thomas met sur le même plan un exemple physique (le feu qui brûle) et un exemple d' action (ma main se lève). Ce sont en effet toutes les opérations naturelles et volontaires qui sont attribuées à Dieu seul par le kalâm.

Thomas connaît la position du kalâm, grâce aux indications données par Maïmonide dans le Guide des égarés, comme en témoigne le De potentia ${ }^{20}$ :

Comme le raconte Rabbi Moïse [Maïmonide], certains théologiens du kalâm (loquentes), selon la loi des Musulmans (Mauri), ont dit que toutes les formes naturelles étaient des accidents. Et puisqu' un accident ne peut passer à un autre sujet, ils pensaient impossible qu' une chose naturelle, par sa forme, induise une forme semblable dans un autre sujet; c' est pourquoi ils disaient que le feu ne chauffe pas, mais que c' est Dieu qui crée la chaleur dans la chose chauffée ${ }^{21}$.

18 Cf. D’Ancona 1995, p. 17: «À la première proposition du De Causis - qui énonce le principe néoplatonicien de la primauté de la cause plus simple - fait pendant, en effet, la proposition 17 - qui distingue la causalité de Dieu-Être pur, en tant que création de l'être de toutes les choses sans aucun présupposé, de celle de l'Intellect, en tant qu' "information" supplémentaire de ce qui a déjà été créé».

19 Thomas d'Aquin, Scriptum super libros Sententiarum II, d. 1, q. 1, a. 4 resp.: Respondeo dicendum, quod circa hanc quaestionem sunt tres positiones. Quarum una est, quod Deus immediate operetur omnia, ita quod nihil aliud est causa alicujus rei; adeo quod dicunt quod ignis non calefacit, sed Deus; nec manus movetur, sed Deus causat ejus motum, et sic de aliis. Sed haec positio stulta est: quia ordinem tollit universi, et propriam operationem a rebus, et destruit judicium sensus. (Je souligne.)

20 Cf. Gilson 1926-1927, p. 5-128, § 1, «La critique thomiste des mutakallemin »; sur l'occasionnalisme médiéval, voir Perler, Rudolph 2000.

21 Thomas d'Aquin, De potentia q. 3, a. 7 resp.: Quidam enim loquentes in lege Maurorum, ut 
Thomas conclut que le kalâm est une position insensée (stulta), car il «supprime l' ordre de l' univers, enlève aux choses leur opération propre, et détruit le jugement des sens». Ces trois griefs sont d'inégale valeur: si Dieu est cohérent, attribuer toute l' efficace des causes à Dieu ne rendrait pas l'univers désordonné. L' objection la plus forte (et qu' on retrouve dans d' autres passages parallèles), est l'importance de donner leur dignité de causes aux causes secondes, c' est-à-dire de leur attribuer une opération propre. La gloire du Créateur est accrue par l' activité de la créature, qui est le miroir où on peut le contempler. Il y a donc plus de grandeur pour Dieu à produire un être qui lui ressemble par son activité (et dans le cas de l'homme, par sa liberté), qu'à créer un simple décor de théâtre où tout est manipulé par un deus ex machina.

Ainsi, sous couvert de préserver la toute-puissance divine, le kalâm va contre la bonté divine, qui tend à se communiquer; c' est pourquoi «les choses ont été faites semblables à Dieu non seulement dans l'être, mais aussi dans l'agir ${ }^{22}$. Sous couvert de toute-puissance, on fait de Dieu un Dieu jaloux de ses prérogatives, qui ne voudrait pas les communiquer aux créatures. Or «Il a voulu conférer aux autres la dignité qu' est l'acte de causer ${ }^{23}$.

\subsection{Contre la création communiquée (le Liber de causis)}

À cette thèse théologique, et même trop théologique, s'oppose la position de «certains des philosophes» (quorumdam philosophorum), qui veulent défendre l'efficace des causes secondes contre le kalâm. Ceux-ci vont trop loin en sens contraire: «Pour soutenir les opérations propres des choses», ses partisans «nient que Dieu ait créé toutes choses immédiatement; mais ils affirment qu' il est immédiatement cause du premier créé ( primi creati), et que celui-ci est cause d' un autre, et ainsi de suite $»^{24}$. En même temps qu' il lui donne l' être, le

Rabbi Moyses narrat, dixerunt, omnes huiusmodi naturales formas accidentia esse: et cum accidens in aliud subiectum transire non possit, impossibile reputabant quod res naturalis per formam suam aliquo modo induceret similem formam in alio subiecto; unde dicebant quod ignis non calefacit, sed Deus creat calorem in re calefacta. Sed si obiiceretur contra eos, quod ex applicatione ignis ad calefactibile, semper sequatur calefactio, nisi per accidens esset aliquid impedimentum igni, quod ostendit ignem esse causam caloris per se; dicebant, quod Deus ita statuit ut iste cursus servaretur in rebus, quod nunquam ipse calorem causaret nisi apposito igne; non quod ignis appositus aliquid ad calefactionem faceret.

Thomas d'Aquin, De potentia, q. 3, a. 7 resp.: ex quo factum est quod res Deo similes fierent non solum in esse, sed etiam in agere.

23 Thomas d'Aquin, Scriptum super libros Sententiarum, II, d. 1, q. 1, a. 4 ad 1: dignitatem causandi aliis conferre voluit.

24 Thomas d'Aquin, Scriptum super libros Sententiarum, II, d. 1, q. 1, a. 4 resp.: Secunda positio est quorumdam philosophorum, qui ut proprias operationes rerum sustineant, Deum immediate omnia creare negant; sed dicunt, quod immediate est causa primi creati, et illud est 
Principe communique à une unique cause seconde sa puissance créatrice, qui produit à son tour la troisième, et ainsi de suite, en une cascade d'émanations. Mais pour Thomas, selon la foi, cette position est erronée: les intelligences, ou plutôt les anges, ne sont pas créateurs, mais Dieu seul est le créateur des réalités visibles et invisibles, des anges et du monde. Mais quel texte philosophique parle ainsi d' une cascade d'émanations et d'un «premier créé»? Quel texte philosophique prétend que le Principe ne produit que l'être, mais non les réalités composées, et que celles-ci sont créées par l'être ${ }^{25}$ ? - La concordance textuelle et doctrinale est sans équivoque : il est clair que Thomas vise précisément le Liber de causis ${ }^{26}$.

Selon saint Thomas, soutenir que Dieu puisse transmettre sa puissance créatrice à une cause seconde, fût-ce l'intelligence, est une position «condamnée au titre d'hérésie» ${ }^{27}$. C' est en effet une sorte d'idolâtrie conceptuelle, qui consiste à attribuer aux créatures un attribut qui n'appartient qu' au Dieu unique (selon la définition de Paul, Romains 1, 25), précisément celui d'être l'unique créateur. Pour Thomas, la création en cascade conduit à renier le monothéisme créationniste. La création est unique et incommunicable. Le pouvoir créateur, c' est-à-dire donateur d' être, ne peut pas être communiqué à la créature. C' est pourquoi la cause première ne doit pas abandonner son pouvoir causal et son efficace au moment même où elle crée la cause seconde.

On ne peut pas davantage se satisfaire de l'idée que Dieu communique tout ce qui est simple, et que les intelligences produisent tout ce qui est composé allusion sans doute au commentaire de la proposition IV du Liber:

causa alterius, et sic deinceps. Sed haec opinio erronea est: quia secundum fidem non ponimus Angelos creatores, sed solum Deum creatorem omnium visibilium et invisibilium.

25 Thomas d'Aquin, Scriptum super libros Sententiarum, II, d. 1, q. 1 a. 3 resp.: in eodem Libro $\langle$ de causis〉 dicitur, quod esse est per creationem, et aliae perfectiones superadditae per informationem, et in compositis praecipue illud esse quod est primae partis, scilicet materiae; et ex parte ista accipiendo creationem, potuit communicari creaturae, ut per virtutem causae primae operantis in ipsa, aliquod esse simplex, vel materia produceretur: et hoc modo philosophi posuerunt intelligentias creare, quamvis sit haereticum.

26 Liber de causis, IV, §44: et esse quidem creatum primum est intelligentia totum («et l'être premier créé est tout entier intelligence», trad. fr. Boulnois et al 1990, p. 45); cf. IV, §37 (Pattin 1966, p. 54): Prima rerum creatarum est esse, et non est ante ipsum creatum aliud («la première des choses créées est l'être, et avant lui rien d' autre n' est créé», trad. fr. Boulnois et al 1990, p. 45).

27 Thomas d'Aquin, Scriptum super libros Sententiarum II, d. 1, q. 1 a. 3 resp.: Quidam enim philosophi posuerunt quod a prima causa immediate est unum primum causatum, a quo postmodum sunt alia, et sic deinceps; unde posuerunt, unam intelligentiam causari mediante alia, et animam mediante intelligentia, et corporalem naturam mediante spirituali; quod pro haeresi condemnatur, quia haec opinio honorum qui Deo debetur, creaturae attribuit; unde propinqua est ad trahendum in idolatriam. 
Et parce que l'intelligence se diversifie en elle (illic), la forme intelligible (intellectibilis) devient diverse. Et de même que des individus d' une multiplicité infinie proviennent d' une forme unique, parce qu' elle se diversifie dans le monde inférieur, de même, des formes intelligibles d'une infinie multiplicité apparaissent à partir de l'être premier créé, parce qu'il se diversifie ${ }^{28}$.

Cette thèse découle de l' affirmation que l' être se transmet aux divers étants par voie de composition et de diversification dans le multiple. Mais là encore, elle suppose que Dieu ne soit pas l'unique créateur, cette fonction étant transmise à sa première créature, l'être.

Saint Thomas, dans son commentaire du Liber, identifie fort justement la source de ce texte avec la Proposition 138 de Proclus: «Parmi tous ceux qui participent à une propriété divine et qui sont divinisés, le tout premier et le plus éminent est l'étant $»^{29}$. Le Livre des causes sous-entend ici qu'il est impossible à la cause première d' agir directement sur des effets concrets sans passer par l'efficace des causes secondes.

La providence de la cause première s'exerce par la nécessité de la nature, et obéit à l' ordre d'une série d'émanations. En cela, le Liber fait écho aux Traités 47 et 48 de Plotin sur la Providence. Plotin interprète la Providence comme l'ordre donné au monde par l'intellect primordial. L' action divine est unique, elle se diversifie au fur et à mesure que l'on pénètre dans ses effets. «Ce monde n' est pas né parce que l'Intelligence a réfléchi qu'il fallait le produire; il résulte d' une nécessité inhérente à la nature de second rang ${ }^{30}$. La raison universelle embrasse toutes choses à la fois, elle ne les crée pas, mais elle coexiste avec elles dans son universalité. Ainsi, les causes immatérielles ne sont jamais les causes immédiates des effets matériels.

28 Liber de causis, IV, $\$ 45$ (Pattin 1966, p. 56): Et quia diversificatur intelligentia, fit illic forma intellectibilis diversa. Et sicut ex forma una, propterea quod diversficatur in mundo inferiori, proveniunt individua infinita in multitudine, similiter ex esse creato primo, propterea quod diversificatur, apparent formae intellectibiles infinitae (trad. fr. Boulnois et al 1990, p. 45 modifiée).

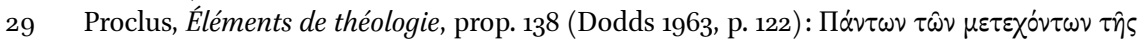

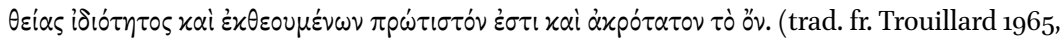
p. 143 modifiée). Thomas d'Aquin, Super Librum de causis expositio: Id autem quod est commune omnibus Intelligentiis distinctis est esse causatum primum de quo praemittit talem propositionem: Prima rerum creatarum est esse, et non est ante ipsum creatum aliud. Et etiam hanc propositionem Proculus in suo libro ponit: omnium participantium divina proprietate et deificatorum primum est et supremum ens.

Plotin, Ennéades III, 2 (47), 2, l. 9-10. 
À leur tour, les Éléments de théologie de Proclus insistent sur la relation entre la cause première et la cause seconde. L'Un ou le Bien est intrinsèquement présent à ce qui émane de lui: l'être et la causalité des causes secondes dépendent de l'être et de la causalité du Bien. Mais la causalité du Bien s' exerce par l' intermédiaire d'une hiérarchie métaphysique: sa causalité ne s' exerce pas directement sur les effets les plus éloignés, mais il agit par l'intermédiaire d'une série de médiations. Si le Bien donne l'être par lui-même, il donne aussi la vie par l' intermédiaire de l'intelligence, et a besoin de la vie pour produire les êtres vivants concrets et existants. Il ne peut donc ni créer ni exercer sa providence sans intermédiaires:

Tout ce qui est divin, à la fois pourvoit aux réalités secondes et n'est rien de celles pour qui il pourvoit, sans que cette providence abaisse son éminence pure et unitaire, ni que cette unité séparée supprime sa providence $^{31}$.

L'Un n' agit pas de manière immédiate sur toutes choses, mais par une cascade d'émanations qui s' engendrent nécessairement.

Pour le Livre des causes, la première cause est agente dans toute action de la cause seconde. Puisque seule la première cause donne l'être, sans elle, il n'y aurait pas d'être des causes secondes, ni d'action de celles-ci. Mais réciproquement, sans les causes secondes, la cause première ne pourrait tout simplement pas agir. Elle agit à travers elles.

Autrement dit, dans leur activité, les causes secondes ne font pas nombre avec la cause première: c' est grâce à l'actualité de la cause première que les causes secondes produisent leurs effets spécifiques. L'action des causes secondes ne s'ajoute pas à un déterminisme divin, elles expriment l'action divine sur le mode qui leur est propre. Cela peut définir en quelque manière la puissance infinie de Dieu, entendue comme un attribut philosophique de Dieu. Cela permet aussi de penser la Providence divine d'une manière philosophique: tout ce qui arrive par l'effet des causes secondes, nécessairement, par accident, ou par la liberté humaine, est d'avance un effet de la causalité divine. La position extrême des philosophes, selon laquelle Dieu ne peut pas agir immédiatement sur toutes choses, peut donc s'appuyer sur le Livre des Causes.

31 Proclus, Éléments de théologie, prop. 122, p. 108 (trad. fr. Trouillard 1965, p. 133, mod.): П人ิv

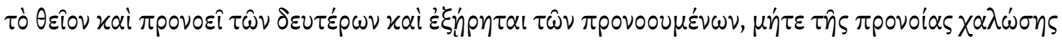

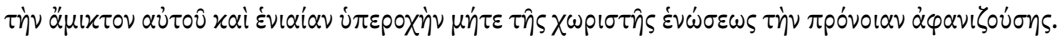




\subsection{Une interprétation théologique du Liber de causis: création immédiate et efficace des causes secondes}

Contre la position folle du kalâm et contre la position extrême de l' émanatisme néoplatonicien, Thomas souhaite élaborer une synthèse rationnelle en harmonie avec la foi, c'est-à-dire une interprétation théologique. Or cette solution du problème, Thomas va encore l' arracher à une discussion serrée du Livre des causes. Il fait donc jouer le deuxième principe (2) du Livre des causes contre les autres:

La créature peut être cause. [...] Cependant, la cause de ces 〈effets〉 est encore Dieu, qui opère en eux 〈les individus〉 plus intimement (magis intime) que les autres causes motrices, parce que lui-même est donateur (dans) de l'être aux choses. [...] C'est pourquoi lui aussi il demeure, ces choses ayant été ôtées, comme il est dit dans le Livre des Causes ${ }^{32}$.

Thomas rappelle que Dieu est donateur d'être immédiat et direct de chaque chose créée. Il n' est pas seulement donateur de leur être, mais même de leur causalité. Magis intime operans est ici un équivalent de plus est influens, mais l'expression met l' accent sur l' intériorité d' une façon qui permet d' harmoniser deux néoplatonismes, celui du Liber avec celui d'Augustin ${ }^{33}$. Dès lors, le premier Principe peut agir sans les causes secondes («ces choses ayant été ôtées»).

La solution est donc de développer une lecture alternative du Liber de causis. Cette lecture permet d' élaborer une synthèse qui rejette les deux positions extrémistes; Dieu peut agir immédiatement en toutes choses, et en même temps, chaque chose possède sa propre opération: «Dieu opère toutes choses immédiatement, et chacune des choses a une opération propre, par laquelle elles sont les causes prochaines des choses, non pas de toutes, mais de certaines ${ }^{34}$. En effet, si la créature ne peut pas en créer une autre, la produire

32 Thomas d'Aquin, Scriptum super libros Sententiarum II, d. 1, q. 1, a. 4 resp.: Aliorum vero quae per motum et generationem producuntur, creatura causa esse potest, vel ita quod habeat causalitatem supra totam speciem, sicut sol est causa in generatione hominis vel leonis; vel ita quod habeat causalitatem ad unum individuum speciei tantum, sicut homo generat hominem, et ignis ignem. Horum tamen causa etiam Deus est, magis intime in eis operans quam aliae causae moventes: quia ipse est dans esse rebus. Causae autem aliae sunt quasi determinantes illud esse. Nullius enim rei totum esse ab aliqua creatura principium sumit, cum materia a Deo solum sit; esse autem est magis intimum cuilibet rei quam ea per quae esse determinatur; unde et remanet, illis remotis, ut in libro de causis dicitur.

Pour Augustin, Dieu est «plus intérieur que l' intime de moi-même» (interior intimo meo, Confessions III, 6, 11 [BA 13, 382]); Madec (Madec 1987-1988) souligne qu' interior est un comparatif: Augustin introduit l'homme à un mouvement vers Dieu (p. 308-309). 
dans l'être, cela veut dire que tout ce qui vient à l'être par une création est créé immédiatement par Dieu. Dieu cause donc immédiatement toutes les opérations dont les créatures sont les causes secondes. Il n' est donc pas possible de distinguer deux parts dans la causalité d'une cause finie: tout ce qu'elle fait, elle le fait par elle-même et par Dieu, de manière indissociable.

Dès lors, Thomas d'Aquin utilise le Liber de causis dans le sens de la toutepuissance divine. Selon l' ordre naturel, la cause seconde produit son effet grâce à la cause première, mais par sa toute-puissance, la cause première peut produire directement l' effet d' une cause seconde sans passer par elle. Et pourtant, même cette doctrine peut se prévaloir du Livre des causes, puisque selon la lettre de celui-ci, la cause première peut agir avant la cause seconde, et que, selon l'interprétation que Thomas en a donné (pour éviter l'émanatisme), elle peut elle-même agir sans la cause seconde ${ }^{35}$. En court-circuitant les causes secondes, le miracle (ici le miracle eucharistique) confirme donc le dispositif métaphysique du Livre des causes. Thomas d' Aquin, en harmonisant la philosophie du Livre des causes avec la théologie de la toute-puissance, rend à la fois possible la création immédiate des causes secondes, et celle des effets sans ces causes. Ce qui n'était dans le Liber qu'une affirmation de la transcendance du Principe devient chez lui une hypothèse contrefactuelle.

Pour saint Thomas, la volonté humaine s' inscrit dans cet ordre global de la nature: certaines causes agissent nécessairement, d' autres par hasard, d' autres encore par un libre arbitre, mais toutes expriment l'action de Dieu.

Parce que la volonté est un principe actif non déterminé à une seule chose, mais qui se rapporte indifféremment à une multiplicité, Dieu la meut de telle manière qu'il ne la détermine pas nécessairement à une seule chose, mais que son mouvement demeure contingent et non nécessaire $^{36}$.

est, quod Deus immediate omnia operatur, et quod res singulae proprias operationes habent, per quas causae proximae rerum sunt, non tamen omnium, sed quorumdam.

35 Thomas d'Aquin, Scriptum super libros Sententiarum IV, d. 12, q. 1, a. 1: sicut dicitur prima propositione Libri de causis "causa prima est vehementioris impressionis supra causatum causae secundae quam ipsa causa secunda” [cf. I, §15; Pattin, p. 49]. Unde quando causa secunda removet influentiam suam a causato, adhuc potest remanere influentia causae primae in causatum illud; sicut remoto rationali, remanet vivum, quo remoto remanet esse. Cum ergo causa prima accidentium et ominium existentium Deus sit; causa autem secunda accidentium sit substantia, quia accidentia ex principiis substantiae causantur; poterit Deus accidentia in esse conservare remota tamen causa secunda, siclicet substantia. Et ideo absque omni dubitatione dicendum est quod Deus potest facere accidens esse sine subiecto.

36 Thomas d'Aquin, Summa theologiae I-II, q. 10, a. 4 resp.: Quia igitur voluntas est activum 
Siger de Brabant soumet cette anlyse à une critique impitoyable. Par-delà la question de la motion de la volonté humaine, c'est plus généralement l'interprétation thomasienne de l'articulation entre causes premières et causes secondes que rejette Siger. Dans son commentaire du Liber de causis, après avoir polémiqué (à la suite de Thomas ${ }^{37}$ ) contre le kalâm, Siger assimile la position de Thomas à la même interprétation sophistique: «C'est pourquoi certains argumentent de manière sophistique ${ }^{38}$. À ses yeux, la position de Thomas, même si elle essaie de s' en démarquer, reste encore marquée parl'incohérence du kalâm; elle en est une forme atténuée, mais non moins erronée. Il faut s' en tenir au schéma émanatiste initial du Liber de causis et refuser l'interprétation maximaliste du principe 2 par Thomas: «la cause primordiale ne peut pas produire l'effet de la cause seconde sans la cause seconde ${ }^{39}$. En effet, si toute cause seconde est créée par le Principe, cela ne veut pas dire qu' elle en reçoit l'opération; la cause seconde reçoit de la cause première l' être, mais pas nécessairement l'agir:

parfois la cause seconde ne reçoit pas son principe d' opération de la cause primordiale, $[\ldots]$ lorsque la forme qui est le principe d'opération et d'où procède l' effet de la cause seconde n' est pas 〈présente $\rangle$ en acte ni en puissance (virtute) dans la cause primordiale ${ }^{40}$.

principium non determinatum ad unum, sed indifferenter se habens ad multa, sic Deus ipsam movet, quod non ex necessitate ad unum determinat, sed remanet motus eius contingens et non necessarius.

37 D. Calma a montré que la critique du kalâm était directement empruntée à la Summa theologiae I, q. 105, a. 5 (Calma 2003, p. 118-135, surtout p. 126-128; Calma 2019); voir déjà Imbach 1996, p. 304-323; et Imbach, Putallaz 1997, p. 166.

38 Siger de Brabant, Quaestiones super Librum de causis, q. 2, p. 41: Unde sophistice quidam $\operatorname{arguunt}(\ldots)$.

39 Siger de Brabant, Quaestiones super Librum de causis, q. 2, p. 40: Causa primaria non potest effectum causae secundariae non potest producere sine causa secundaria. Dans les Quaestiones in libros Meteorum (éd. Duin 1954, p. 111), il définissait la cause première de manière clairement émanatiste: in qua est tota virtus productiva alicuius effectus, non tamen secundum quod est productiva, sed per modum excellentiorem; non oportet quod immediate regat ipsum.

40 Siger de Brabant, Quaestiones super Librum de causis, q. 2, p. 40-41: aliquando formam quae est operationis principium non accipit a primaria [...]; cum tunc in primaria non sit actu nec virtute forma, quae est operationis principium, a qua procedit effectus causae secundariae. Sur ce point, voir les remarques de Calma 2019, p. 268-300, notamment p. 273: «Les causes secondaires reçoivent toutes les qualités de la cause première, mais cela n'enlève rien au fait qu' elles ont leurs propres opérations, leurs propres vertus et exercent leur influence sur leurs effets, en dépit du fait que la cause première agit d'une manière plus noble et supérieure». 
Dans ce cas, il n' est pas nécessaire que la cause première puisse produire son effet sans la cause seconde ${ }^{41}$ : elle n' intervient pas directement dans le monde sublunaire, mais elle a précisément besoin de la cause seconde comme d'un instrument qui spécifie son action sur un objet particulier, et sans passer par celle-ci, elle ne pourrait pas descendre à une telle particularité. Par définition, la forme de la cause première est plus excellente que celle de la cause seconde, son principe d' opération est donc par nature plus excellent, et il ne peut pas s'y réduire ou s'y abaisser. «C' est pourquoi l' effet de la cause seconde ne pourra pas être produit immédiatement par la cause primordiale ${ }^{42}$. Siger s' attaque alors à l'explication thomasienne de l'eucharistie: s' agissant de la conversion de la substance du pain, certains croient à tort pouvoir expliquer par «la raison naturelle» (naturali ratione) la mutation de la substance et le maintien des accidents. Ils soutiennent (comme Thomas) que, «puisque la cause première est la cause de toutes les causes intermédiaires entre elle-même et l' accident, elle peut faire à elle seule que [...] l' accident subsiste sans la substance $»^{43}$. Mais puisque la cause première ne peut pas agir sans être spécifiée par la forme et l'efficace propre des causes secondes, une telle explication est de part en part erronée.

Il faut donc comprendre que la cause seconde reçoit de la cause première son être et sa nature, «qui est le principe de son opération», mais qu'elle n'en reçoit pas l'opération elle-même. Précisément, sa forme propre définit sa manière d'agir, mais elle s'ajoute à la causalité primordiale et l'applique à son effet particulier. On ne peut donc pas dire que «la cause primordiale seule peut tout ce que peut la cause première jointe à la seconde $»^{44}$. Dans son action, la cause seconde ajoute quelque chose à la première, son activité n' est

41 Siger de Brabant, Quaestiones super Librum de causis, q. 2, p. 40: et tunc non oportet quod causa primaria possit effectum producere sine causa secundaria.

42 Siger de Brabant, Quaestiones super Librum de causis, q. 2, p. 41: tunc effectus causae secundariae non poterit produci immediate a causa primaria. (je souligne.)

43 Siger de Brabant, Quaestiones super Librum de causis, q. 2, p. 41: Quidam arguunt credentes naturali ratione ostendere et demonstrare quod causa prima possit facere quod accidens existat sine subiecto illius accidentis, propter hoc quod causa prima est cause omnium causarum mediarum accidentis inter ipsam et accidens, et ideo sola facere possit quod existat accidens, [...] poterit facere ut sine substantia subsistat accidens. Siger souligne qu' il croit lui aussi à la conversion eucharistie; mais il vaut mieux admettre une telle proposition par la foi et non comme résultant d' une démonstration, plutôt que la soutenir comme la conclusion d' une démonstration fausse (est enim oratio conclusa peior seipsa non conclusa, ibid.).

44 Siger de Brabant, Quaestiones super Librum de causis, q. 2, p. 41: Quamquam enim causa secundaria substantiam et naturam, quae est operationis principium, accipiat a primaria, non tamen oportet quod primaria sola possit quidquid potest primaria cum secundaria. 
pas toute entière contenue dans la première. Les deux causes s'additionnent. Elles font nombre. Elles concourent au même effet.

Siger met ainsi en place un tout autre modèle d'articulation entre la cause première et la cause seconde, qui conserve la position initiale du Liber de causis (impossibilité d' une activité immédiate du Principe) et rejette l'interprétation de Thomas (les causes secondes reçoivent de la première leur être mais non leur opération). Et pourtant, ce type d'articulation entre les causes n'est pas seulement un retour à la théorie émanatiste du néoplatonisme; il peut aussi être considéré comme la préfiguration d'une nouvelle articulation des causes, qui donnera naissance au second modèle d'interprétation du Liber causis.

\section{La toute-puissance et les causes partielles concourantes chez Duns} Scot

\subsection{Le problème du mal}

La continuité causale entre Dieu et l'action humaine, médiatisée par la théorie des causes secondes, semble en effet rendre Dieu auteur du mal, donc cause et responsable de celui-ci. En effet, le Liber de causis fournit la majeure d'un inquiétant syllogisme: «toute opération que produit la cause seconde, la cause première le produit aussi $»^{45}$; or notre volonté est une cause seconde qui produit le mal; celui-ci semble donc provenir de Dieu, qui est cette cause première ${ }^{46}$. Le commentaire de Roger Bacon au Livre des causes le dit à sa manière: «Tout ce qui est cause d'une cause est cause de son effet; or la cause primordiale est la cause de la cause seconde; donc elle cause aussi [son effet] $\gg^{47}$. Puisque la volonté est une cause seconde, Dieu est la cause de la volonté, or la volonté est cause du mal, donc Dieu est cause du mal.

La solution scotiste de cette difficulté est peu développée. Scot se contente de soutenir que trois réponses sont possibles. 1. La volonté finie a pu com-

45 Le Liber de causis I, §14: Omnem operationem quam causa efficit secunda, prima etiam causa efficit (Pattin 1966, p. 49, trad. fr. Boulnois et al 199o, p. 41).

46 Duns Scot, Lectura II, d. 34-37, q. 4, §17 (XIX, p. 328): Utrum peccatum possit esse a Deo?: Quod sic, videtur: "Quod est a causa inferiore, est a causa superiore"; sed peccatum est a voluntate; ergo est a Deo, qui est causa superior.

47 Roger Bacon, Quaestiones supra Librum de causis, q. [11] in prop. 1 (éd. Steele 1935, p. 10): Quidquid est causa causae, est causa causati; sed causa primaria est causa secundae causae; quare et causat. Chez Duns Scot, l' argument devient: Confirmatur per illam propositionem: "Quod est causa causae, est causa causati"; Deus est causa voluntatis, voluntas est causa peccati; igitur etc. (Lectura II, d. 37, q. 4, §17; XIX, p. 328). 
mettre le mal parce qu' elle était par nature finie, mêlée d'être et de néant, donc faillible (defectibilis), mais Dieu n'est pas responsable de sa défaillance factuelle. Ainsi le bien n' est pas une cause positive, efficiente, mais une cause déficiente du mal. 2. La volonté a pu vouloir le bien comme cause positive, mais défaillir par accident, et choisir le mal, ce qui la rapproche du cas précédent. 3 . La volonté a pu vouloir le bien comme cause positive, mais s' être méprise sur ses caractéristiques qui s' avèrent en fait un $\mathrm{mal}^{48}$.

Quoi qu' il en soit, il semble évident que c' est l' implication intime de l' action divine au cour de l'action humaine (c'est-à-dire le théorème du Livre des causes) qui menace la bonté divine. Il fallait donc trouver un moyen de dissocier l'un et l' autre.

\subsection{La possibilité de l'immédiateté}

Nous l'avons vu, le Liber de causis soumettait totalement la Providence divine à l' ordre du monde, si bien qu' il n' existait pas d' autre cours possible du monde que celui qui déployait l'influx divin dans l' ordre médiat des causes secondes.

Au nom du concept de toute-puissance, Duns Scot, au contraire, critique sévèrement la cosmologie du Livre des causes. En effet, on peut comprendre la toute-puissance en un sens philosophique, et «tout-puissant» signifie celui qui peut tout le possible, médiatement ou immédiatement, en tant que cause prochaine ou éloignée. C' est exactement la cosmologie du Liber de causis. - Ou bien «tout-puissant» est pris au sens propre, théologique, c' est-à-dire au sens où la cause première peut produire tout effet immédiatement, «sans aucune coopération d' une autre cause agente ${ }^{49}$. Autrement dit, Scot emboîte le pas à l'interprétation thomasienne du principe 2 : le premier Principe peut agir sans la cause seconde. Il rejette l'interprétation sigérienne (d'ailleurs condamnée en $\left.1277^{50}\right)$.

Il faut donc soutenir: «tout ce que peut la cause efficiente première avec la cause seconde, elle le peut par elle-même immédiatement ${ }^{51}$. Cette proposition est indémontrable philosophiquement, mais elle est précisément ce

48 Duns Scot, Lectura II, d. 34-37, q. 4, §. 98-101 (XIX, p. 350-351).

49 Duns Scot, Ordinatio I, d. 42, q. 1, §.8 (VI, 343). Sur la place du Liber de causis dans l' œuvre de Scot, voir Counet 2019, p. 251-267.

50 La condamnation parisienne de 1277, Art. 199, p. 140-141: Quod in causis efficientibus, cessante prima non cessat secunda ab operatione sua, dum tamen secunda operetur secundum naturam suam. - «Dans les causes efficientes, si la première cesse d'agir, la seconde ne cesse pas pour autant son opération propre, pourvu toutefois que la seconde opère selon sa nature».

$5^{1}$ Duns Scot, Ordinatio I, d. 42, q. 1, §14 (VI, 346): quidquid potest causa effectiva prima cum causa secunda, potest per se immediate. 
qu' exige la foi en un Dieu tout-puissant et créateur. C' est ce qu'Ockham appellera la «proposition fameuse des théologiens». Chez Ockham, elle s'exprime ainsi: «tout ce que Dieu produit par la médiation des causes secondes, il peut le produire et le conserver sans elles $»^{52}$. C'est une proposition théologique, comme telle indémontrable par la seule raison, car elle découle de la profession de foi en la toute-puissance divine ${ }^{53}$.

C'est pourquoi, selon Duns Scot, la hiérarchie entre une cause première et une cause seconde ne suffit pas à expliquer la spécificité de l'action humaine. Ainsi, il n'est pas du tout nécessaire de maintenir l'antériorité de l'efficace de la cause première sur l'efficace de la cause seconde (principe 3 du Livre des causes). On peut en effet penser son action par analogie avec celle du cœur, principe premier du mouvement des êtres vivants, et de la main, principe second de l'action d'écrire:

Je concède que, lorsqu' il y a deux causes ordonnées, toutes deux causent l' effet, - mais elles le font différemment, car la cause supérieure cause davantage [= principe 1]; ainsi, si le cœur est la cause supérieure et la main la cause inférieure de l'écriture, l'une et l' autre (tant le cœur que la main) sont causes; cependant, ce n' est pas le cœur qui produit l'écriture avant la main ${ }^{54}$.

Selon cette analogie, on ne peut pas dire que la cause première produit l'être, puis que la cause seconde détermine l' action. Il n'y a donc pas d'antériorité de la cause première sur la cause seconde, mais une collaboration dans une même action, même si la première est supérieure à l' autre.

$5^{2}$ Ockham, Quodlibet vI, q. 6 (Opera theologica IX, 604): In illo articulo [la toute-puissance divine, premier article du credo] fundatur ila propositio famosa theologorum: 'quidquid Deus producit mediantibus causis secundis, potest immediate sine illis producere et conservare'; cf. q. 1 (IX, 587); Ordinatio II, d. 42, q. 1, §14 (v, 69); et les analyses d'A. de Muralt $1993^{2}$, p. 332-334.

53 Cf. Vignaux 1948, p. 22: «Nous connaissions à la dialectique de potentia absoluta une fonction religieuse. Celle-ci consistait, en montrant la contingence de tout l'ordre de la nature et de la grâce, à rappeler la dépendance de toutes choses à l'égard d'un Principe qui agit avec une liberté, une gratuité souveraine. À un nouvel examen des textes, la même argumentation de théologiens paraît résoudre une difficulté toute philosophique: le discernement, dans l' objet d' une investigation, des caractères qu'il peut laisser tomber et de ceux qu'il ne saurait perdre sans cesser d'être lui-même».

54 Duns Scot, Ordinatio I, d. 36, §65 (VI, 297): concedo quod quando sunt duae causae ordinatae, ambae causant effectum, - et aliter, quia superior plus causat; et ita si cor est causa superior et manus inferior respectu litterarum, utraque causat (tam cor quam manus), non tamen prius cor quam manus producit litteras. 
Il faut donc redéfinir la relation entre cause première et cause seconde en éliminant le principe 3 du Livre des causes, et en le remplaçant par une théorie des causes concourant ensemble au même effet, mais sans antériorité de l'une sur l'autre, c'est-à-dire sans que l'une déclenche l'autre.

La position de Scot gravite donc autour de trois thèses:

1. La cause première cause davantage que la cause seconde (principe 1 );

2. La cause première peut causer sans la cause seconde (interprétation théologique du principe 2);

3. Mais elle ne cause pas avant la cause seconde (négation du principe 3).

\subsection{La réforme de la théorie de la causalité}

Duns Scot reconstruit alors le concept de causalité pour lui-même. Pour cela, il opère une alliance entre la théorie sigérienne de la causalité (la cause seconde s'ajoute à la cause première sans être précontenue en elle), et l' exigence théologique de toute-puissance (acquise dès saint Thomas: la cause première peut agir sans la cause seconde, principe 2 du Livre des causes).

En effet, Duns Scot définit la causalité dans le cadre d' une théorie de l' induction. Même si personne ne peut avoir l'expérience de tous les cas particuliers, l'homme d'expérience accède à une science infaillible de ce qui est universel: cette science est qualitativement différente ${ }^{55}$. Ainsi, nous admettons comme évidente une formulation du principe de causalité: tout ce qui provient régulièrement d' autre chose en est l' effet et celle-ci en est la cause. Nous pouvons savoir qu'il en va ainsi toujours et universellement, parce que nous pouvons attribuer ces effets à une cause naturelle et non libre, et expliquer les exceptions par des conjonctions accidentelles qui s' ajoutent à ces natures. Quelles que soient les variations accidentelles, l' effet provient de la nature, c' est-à-dire de l' essence d' une cause.

Remarquons que l'analyse de Scot exclut les causes libres. Cela s' explique par le fait que celles-ci se rangent (aux côtés du hasard) dans la catégorie des causes qui produisent aussi bien un effet que le contraire. Duns Scot refonde ainsi le triptyque aristotélicien des causes produisant le plus souvent, le plus

55 Duns Scot, Ordinatio I, d. 3, §. 235: licet experientia non habeatur de omnibus singularibus sed de pluribus, neque quod semper sed quod pluries, tamen expertus infallibiliter novit quia ita est, et semper et in omnibus - et hoc per istam propositionem quiescentem in anima: 'quicquid evenit ut in pluribus ab aliqua causa non libera, est effectus naturalis illius causae. [...] per naturam ipsam in se, sequitur talis effectus (éd. Vat. III, 141-142); trad. Boulnois 1988, p. 181. (Je souligne.) 
rarement, ou également leur effet ${ }^{56}$. Mais cette analyse a une conséquence: la volonté, cause libre, ne relève pas du principe de causalité. Le libre arbitre n' appartient pas au domaine des objets régis par ce principe.

\subsection{La cause du vouloir}

Duns Scot s' interroge alors sur la relation entre la causalité et la liberté. Quelle est la cause qui nous fait vouloir? Il faut d' abord observer que l' examen par Scot de la cause du vouloir est analogue, dans sa structure, à son examen de la cause de l'intellection. Dans le cas de l' intellection, ce n' est ni l' objet, ni l'âme seule qui est la cause totale de l'intellection. Il en découle que tous deux forment ensemble une seule cause intégrale (una causa integra) à l' égard de la connaissance engendrée en nous ${ }^{57}$. Il en va de même pour l' objet voulu et la volonté: ni l'objet ni la volonté ne sont la cause totale du vouloir; mais notre volonté a besoin au minimum d'un objet; celui-ci est donc la cause partielle de son vouloir.

Comment articuler ces deux causes entre elles? Plusieurs possibilités s' offrent à nous pour penser le concours des causes:

Ou bien [1] les causes agissent à égalité: c'est une addition accidentelle de deux forces;

Ou bien [2] les causes agissent dans l'inégalité, mais selon un ordre essentiel:

Mais alors, ou bien [2.a] la cause supérieure meut l'inférieure, et alors deux cas sont possibles:

Soit $\left[2 . a_{1}\right]$ la cause inférieure reçoit la puissance et la forme de son mouvement (par exemple, le soleil et le père dans la génération, comme l'enseigne Aristote, Physique II, 2, 194b13);

Soit $\left[2 . \mathrm{a}_{2}\right]$ la cause supérieure donne la puissance, mais non la forme (Ce cas correspond à ce que Thomas appelait une cause instrumentale $\left.{ }^{58}\right)$.

Ou bien [2.b] la cause supérieure ne meut pas l' inférieure, et ne lui donne

56 Aristote, De l'interprétation ch. 9, 19a18-22.

57 Duns Scot, Ordinatio I, d. 3, § 494 (III, 292).

$5^{8}$ Thomas d' Aquin, Summa contra Gentiles III, 70, § 2464: superius agens dat virtutem ipsam inferiori agenti per quam agit; vel conservat eam; aut etiam applicat eam ad agendum, sicut artifex applicat instrumentum ad proprium effectum; cui tamen non dat formam per quam agit instrumentum, nec conservat, sed dat ei solum motum. Duns Scot le rapproche du bâton mû par la main humaine (cf. Aristote, Physique viII, 5, 256a 30). 
pas sa puissance, mais elle s'y ajoute (dans la génération, la puissance du père collabore avec celle de la mère, la femme n' est pas simplement passive, - selon Galien contre Aristote) ${ }^{59}$.

Considérons l' ordre essentiel entre la cause première et la cause seconde. Les causes partielles possèdent leur puissance active séparément, mais elles produisent leur effet ensemble. Au lieu de la théorie simple du Livre des causes, qui articule directement cause première et cause seconde, Duns Scot introduit une démultiplication des types de causes. Dès lors, il peut opérer une double mutation:

1. Dissocier la puissance causale de la forme: dans le cas du bâton frappant une balle (de golf?), le principe donateur de forme n'est pas celui qui donne la puissance causale.

2. Soutenir que la puissance causale inférieure, loin d'être précontenue par éminence dans la cause supérieure, comme c'était le cas chez Proclus, dans le Livre des causes et chez Denys, peut lui ajouter une perfection qu' elle n'a pas. C'était précisément l' interprétation suggérée par Siger.

Dès lors, on ne peut plus dire que la cause seconde exprime la causalité de la cause première. Il faut dire qu' elle y ajoute une puissance supplémentaire. Une telle analyse est profondément contraire à la logique néoplatonicienne de l'éminence, pour laquelle ajouter de l' imperfection, c' est amoindrir la perfection. Or dans l'espace des causes partielles ouvert par Scot, la cause seconde ne participe pas à la causalité de la cause première, elle s'y ajoute: ajouter de l'imperfection, c'est encore ajouter de la perfection.

59 Duns Scot, Ordinatio I, d. 3, § 495-496 (III, 293-294): distinguo de pluribus causis concurrentibus ad eundem effectum. [1] Quaedam enim ex æquo concurrunt, sicut duo trahentes aliquod idem corpus. [2] Quaedam non ex cequo, sed habentes ordinem essentialem, et hoc dupliciter: $[a]$ vel sic quod superior moveat inferiorem, ita quod inferior non agit nisi quia mota ex superiore, et $\left[a_{1}\right]$ quandoque causa talis inferior habet a superiore virtutem illam seu formam qua movet, $\left[\mathrm{a}_{2}\right]$ quandoque non, sed formam ab alio, et a causa superiore solam motionem actualem, ad producendum effectum; $[b]$ quandoque autem superior non movet inferiorem, nec dat ei virtutem qua movet, sed superior de se habet virtutem perfectiorem agendi, et inferior habet virtutem imperfectiorem agendi. Exemplus primi membri huius divisionis: de potentia motiva quae est in manu, et baculo, et pila $\left[=\mathrm{a}_{2}\right]$; exemplum secundi: simater ponatur habere virtutem activam in generatione prolis, illa et potentia patris concurrunt ut duae potentiae partiales, ordinata quidem, quia altera perfectior reliqua [= b]; non tamen imperfectior recipit suam causalitatem a causa perfectiore, nec tota illa causalitas est eminenter in causa perfectiore, sed aliquid addit causa imperfectior, in tantem quod effectus potest esse perfectior a causa perfectiore et imperfectiore quam a sola causa perfectiore. 
Par là, c'est toute la cosmologie néoplatonicienne qui se trouve profondément subvertie: la cause partielle inférieure n'agit plus «en vertu de la cause supérieure ${ }^{60}$. Un étant inférieur peut avoir sa forme et sa puissance par lui-même, sans dépendre d'une cause supérieure pour agir, et ajouter sa propre perfection à celle de la cause première. Le concept de causes ordonnées n' implique plus que la cause seconde agisse en vertu de la première, c' est-àdire qu' elle en reçoive la forme par laquelle elle agit. Elle a acquis une forme d'autonomie.

Ces causes partielles concourantes remplissent ainsi trois conditions:

1. Elles sont de nature différente: aucune ne peut remplacer l'autre en accroissant l'intensité de sa propre causalité.

2. Elles sont simultanées: il est nécessaire, pour que l'effet total soit produit, que chacune d'elles soit présente.

3. L'une est plus parfaite que l'autre: sans elle, la moins parfaite ne peut pas agir, même si, lorsqu' elle agit, elle le fait selon sa nature et son efficace propre. Les deux causes font nombre, et leur efficace s' additionne.

Il s' agit là d'une révolution conceptuelle, dont peu de commentateurs ont vu l'importance ${ }^{61}$. Car le concept de concursus impose de distinguer deux parts, alors que Thomas d'Aquin les estimait inséparables ${ }^{62}$. Il peut recouvrir tantôt une coopération, une synergie, tantôt une concurrence, un antagonisme

Contre Thomas, Scot fait également jouer l' idée que la cause première agit de manière contingente. - En effet, admettons que, selon le principe 3 du Livre des causes, la cause première agisse avant la cause seconde; si la première meut de manière nécessaire, lorsqu' elle agit, elle produit nécessairement la cause seconde et sa causalité. Par conséquent, si elle agit de manière nécessaire, la cause seconde est déjà mue par elle, et elle est déjà nécessairement

6o Duns Scot, Ordinatio II, d. 9, q. 2, §118-119 (VIII, 192-193): Dico quod agere in virtute b potest intelligi dupliciter: vel quod a recipiat formam ab ipso b, qua agat, vel quod-habita forma-, recipiat ab ipso ipsam actionem. Secundo autem modo, causa efficiens non agit in virtute alterius, nam ignos - habens formam activam qua agit - non recipit actionem calefaciendi a sole sive specialem motionem ad calefaciendum [... §119] nec etiam hoc est de ratione causarum ordinatarum agentium, quod scilicet una agat in virtute alterius; sed sufficit quod una principalius agat quam alia.

61 À l'exception d'A. de Muralt 1993: «la seule révolution doctrinale digne de ce nom qui se soit produite dans l'histoire de la pensée occidentale» (p. 118; cf. p. XIII, «révolution silencieuse»; p. 258, «la révolution structurelle la plus considérable que la philosophie médiévale ait opérée»).

62 Thomas d'Aquin, Summa contra Gentiles III, 70, § 2466: Non sic idem effectus causae naturali et divinae virtuti attribuitur quasi partim a Deo, et partim a naturali agente fiat, sed totus ab utroque. 
déterminée: «dans ce moment antérieur, si la cause donnait à cet effet un être nécessaire, la seconde cause ne serait jamais capable de donner un être contingent au même effet» ${ }^{63}$. Et même si nous rejetons le principe 3, et soutenons que la seconde cause agit en même temps que la première, cette cause seconde ne sera jamais capable d' entraver la causalité divine. Elle ne pourra pas changer le statut modal de l'effet, et rendre contingent ce qui était déjà nécessaire. Donc, quel que soit notre rapport au principe 3, si Dieu crée nécessairement, tout sera nécessaire. - De surcroît, si la cause première causait nécessairement, elle causerait immédiatement tout ce qu' elle peut causer. Elle causerait tout le possible, tout ne ferait qu' un, et tout serait parfait, voire infini ${ }^{64}$. - Ce sont pour Scot deux arguments par l'absurde, car la contingence est attestée dans le cas des actions libres et la finitude dans le cas du monde. Or si la cause première n' agissait pas d' emblée de manière contingente, aucune cause ne pourrait agir de manière contingente ${ }^{65}$. Il faut donc admettre la contingence dès le départ, au cœur de la volonté divine, positivement, et non comme une indétermination négative, qui proviendrait des flottements dans la causalité naturelle et de l'imperfection de la matière ${ }^{66}$.

Il faut donc identifier la cause première avec une volonté libre. Celle-ci n'a donc rien à voir avec une émanation nécessaire du premier Principe. C' est aussi ce qui permet à la volonté finie d'être une cause contingente et libre.

63 Duns Scot, Ordinatio I, d. 8, § 287 (IV, 315): causa prior prius naturaliter respicit causatum quam causa posterior, ex prima propositione De causis; ergo in illo priore, si totaliter causat, tunc causat totum illud quod in secundo signo deberet a causa secunda causari. [...] \$. 289: causabit et omnia et unum solum, ita quod omnia erunt tantum unum (IV, 317). Cf. Duns Scot, Lectura I, d. 39, §36: Praeterea, prima causa prius comparatur ad effectum quam causa secunda, sicut patet per primam propositionem De causis; si igitur in illo priore causa daret esse necessarium effectui, tunc secunda causa non posset dare aliquod esse contingenter illi effectui, quia tunc effectus haberet esse oppositis modis (necessario et contingenter). Si autem dicatur quod simul causa prima et proxima dent esse effectui, non potest adhuc causa secunda dare esse contingenter effectui si causa prima det esse necessario, quia non potest esse quod idem effectus secundum suum esse habeat necessariam habitudinem ad causam perfectam, et contingentem ad causam imperfectam (quia si necessariam habet habitudinem ad causam perfectam, igitur si causa contingens non sit, adhuc effectus habebit esse; igitur non capit esse a causa contingenti). - Scot cite ici notre «principe 2 » dans le commentaire de la proposition I (Liber de causis I, §3).

64 Duns Scot, Ordinatio I, d. 8, §284 (perfection), §. 288 (infinité), §.289 (unité et totalité) (IV, 315-317). Ainsi, c' est parce que Dieu est libre qu' il y a du mal dans le monde: «il y a du mal dans l'univers, donc Dieu ne cause pas nécessairement» (\$. 283; IV, 314).

65 Duns Scot, Reportatio Parisiensis II, d. 1, q. 3, § [12] (éd. Vivès 1891, t. 22, p. 537).

66 Duns Scot, Ordinatio I, d. 2, §8o (II, 176-177), cf. les §81-88 (II, 177-180). Raisonnement parallèle en I, d. 8, §282 (IV, 313-314). 


\section{$4 \quad$ Ockham et l' articulation des causes partielles concourantes}

Selon Ockham, Dieu et l'homme collaborent («co-agissent») comme deux causes partielles concourantes. Ockham parle alors d'influence générale:

Dieu est cause partielle, qui concourt immédiatement avec toutes les causes à produire n' importe quel effet, et cela, c' est sans doute concourir selon une influence générale ${ }^{67}$.

Le concept d' influentia generalis est déjà présent dans l'école franciscaine, et notamment chez Bonaventure; il désigne seulement l' action du Principe qui soutient les causes secondes dans leur être et dans leur activité, mais sans déterminer leur action par une intervention spéciale ${ }^{68}$. Pour qu' une cause seconde opère, il faut donc qu' elle ajoute son influx spécifique et déterminé à la puissance active en général qui lui est communiquée par Dieu.

Le raisonnement d'Ockham part de la liberté des êtres spirituels, l'homme et l' ange. Dans le cas d' un vouloir fini, la volonté et l' objet sont les deux causes partielles de l'acte de vouloir; puisque la volonté est libre, même si la volonté et l'objet sont approchés l'un de l' autre, il suffit que la volonté ne le veuille pas pour que l'effet, le vouloir, ne s'ensuive pas. La volonté a en quelque sorte un droit de veto. Il en va de même, dans le rapport entre Dieu et la volonté finie, sauf que cette fois-ci, les deux causes partielles (Dieu et la volonté) ont chacune un droit de veto. Si soit Dieu soit la volonté ne le veulent pas, il ne peut pas y avoir de vouloir déclenché par la volonté. Et en même temps, Dieu lui-même ne peut pas forcer le consentement de l' autre cause partielle: «Il est donc impossible qu'il y ait un acte dans la volonté qui ne soit pas en son pouvoir» ${ }^{69}$. La part de l'homme est l' acte de vouloir; celui-ci est libre et indépendant de Dieu. C' est seulement si par sa puissance absolue, Dieu devenait la cause totale de l'action qu'une volition pourrait être causée en nous sans que notre volonté soit active ${ }^{70}$. Mais selon la puissance ordonnée de Dieu, c' est-à-dire selon le cours normal du monde, notre volonté est devenue une citadelle inexpugnable,

67 Ockham, Quaestiones variae q. 5, p. 162-163: Deus est causa partialis immediate concurrens cum omni causa ad producendum quemcumque effectum, et hoc est forte concurrere secundum generalem influentiam.

68 Cf. Dhont 1947; Schmutz 200o, p. 217-264, notamment p. 230. Voir déjà Bonaventure, De scientia Christi, q. 4 (Opera Omnia, v, p. 23 a): Illa lucis influentia aut est generalis quantum deus influit in omnibus creaturis, aut est specialis sicut deus influit per gratiam.

69 Ockham, Ordinatio II, q. 15 (v, 347).

$70 \quad$ Ockham, Ordinatio II, q. 15 (v, 351). 
et la volonté de Dieu apparait comme une cause extérieure, générale, qui ne meut pas spécifiquement notre volonté à vouloir de l'intérieur. Elle ne risque donc plus de la déterminer.

Le modèle proposé par Ockham (précisément en discutant le premier théorème du Liber de causis ${ }^{71}$ ) est remarquable. Dieu agit avec les causes secondes, et non plus par leur intermédiaire. La cause principale, Dieu, est comparée à un homme fort (capable de porter dix unités). La cause secondaire, l' homme, est comme un homme faible (capable de porter une unité): il ne peut qu' aider à porter ce poids, alors que l'homme très fort n' en a pas besoin. Mais ensemble, ils produisent un effet plus intense que séparément. De même, Dieu «ne veut pas tout produire seul, mais il co-agit (coagit) avec les causes secondes en tant que cause partielle, bien qu'il soit la cause principale ${ }^{72}$. Le concept de co-action est éloquent: Ockham n'affirme plus qu'une cause est dépendante de l' autre (qui cause son activité), mais qu' elles sont l'une et l' autre du même ordre, responsables (causes) chacune d'une partie de l'action. Dieu et la volonté sont mis sur le même plan. Ainsi, «les causes secondes ne sont pas superflues, car Dieu n' agit pas dans toute action selon toute sa puissance $\gg^{73}$. C'est parce que Dieu tempère son action qu'il laisse une place à l'activité humaine. Mais dans son déclenchement, celle-ci est devenue indépendante.

Certes, Ockham nous signale que la comparaison avec le modèle humain est grossière (rude). Mais est-il conscient de ce à quoi l' engage ce modèle? Même en envisageant que le fini s' ajoute à l'infini, que la puissance finie de l' homme puisse ajouter quelque chose à la puissance infinie de Dieu, le trait saillant de cette théorie des causes partielles concourantes est précisément qu' elle per-

71 Ockham, Ordinatio II, q. 3-4 (v, 71): dicitur quod Deus non potest esse causa cuiuslibet prima prioritate durationis, quia "causa prima plus influit et prius quam causa secunda", etc. (ce qui reprend les principes 1 et 3). Ockham, Ordinatio II, q. 3-4 (v, 72): licet Deus agat mediantibus causis secundis vel magis cum eis, non dicitur Deus mediate agere, nec secundae causae frustra, cum sit agens voluntarium, non necessarium. Et si esset agens necessarium, adhuc ageret immediate. Exemplum rude ad hoc: ponatur quod unus homo fortissimus possit portare decem per se, et nullus alius posset portare sine eo. Tunc si aliquis debilis portet illa decem cum illo forti, nihilominus ille fortis dicetur immediate portare sicut debilis. Nec propter hoc superfluit ille debilis sifortis non vult per se portare decem. Sic est in proposito. Deus enim est tale agens quod potest esse causa totalis effectus sine quocumque alio.

72 Ockham, Ordinatio II, q. 3-4 (v, 72): Quia tamen "Deus sic res administrat ut eas habere motus proprios sinat", secundum Augustinum [De Civitate Dei VII, 30], non vult solus totum producere, sed coagit cum causis secundis tamquam causa partialis, licet sit principalior. Ita quod ipse est causa immediata omnium quando agit cum causis secundis sicut quando agit sine illis.

73 Ockham, Ordinatio II, q. 3-4: Nec propter hoc superfluunt causae secundae, quia Deus non agit in qualibet actione secundum totam potentiam suam. 
met de penser le rapport entre Dieu et l' homme tantôt comme une aide, tantôt comme une concurrence. C' est clairement la conséquence du principe ockhamien: la volonté humaine, comme la volonté divine, est sans cause. Il n'y a plus de cause du vouloir humain, pas même Dieu. Par conséquent, le vouloir humain ne peut pas dépendre de Dieu, il ne peut que collaborer avec lui ${ }^{74}$.

L'acte de vouloir est d'un autre ordre que la causalité naturelle: la volonté est la seule à pouvoir causer ou ne pas causer son effet; et de ce fait, elle est la seule à ne pas pouvoir être produite par Dieu. La volonté est souveraine, auto-déterminée. Dans sa toute-puissance ordonnée, Dieu lui-même ne peut pas m' empêcher de vouloir. Moi seul puis vouloir, et même Dieu ne peut qu'y collaborer. Sur le plan de la liberté de la volonté, l' homme est devenu l'égal de Dieu $^{75}$.

Par rapport à Thomas d'Aquin, la mutation est grande. Pour Thomas, la cause première ne pouvait pas s'additionner avec quelque cause que ce soit; elle les précontenait toutes, et agissait à travers elles. Dieu agissait à travers la liberté humaine, mais réciproquement, dans sa puissance ordonnée, la volonté divine ne pouvait rien sans l'homme. Pour Ockham, ce sont deux volontés qui peuvent s' additionner en collaborant, ou se soustraire en s'opposant. L' autonomie peut devenir une résistance.

La seconde caractéristique de cette thèse est de renouer avec l'occasionnalisme: «on ne peut pas démontrer qu'un effet est produit par une cause seconde», et aucune action humaine ne peut nous prouver que son agent est un homme ${ }^{76}$. La relation de cause à effet peut n'être qu' une régularité apparente provenant de la puissance ordonnée de Dieu. En réalité, rien ne prouve

74 Ockham, Quodlibet III, 14: omnis actus alius a voluntate potest fieri a solo Deo (IX, 254).

75 Ockham est-il un «nouveau pélagien», comme le supposent ses adversaires? Pour pouvoir soutenir cela, il faudrait comprendre que l' homme a le pouvoir de susciter le concours de Dieu. Mais rien chez Ockham ne permet d' affirmer cela. Au contraire, la volonté de Dieu reste libre de concourir ou non à l' action de la cause finie. C' est d' ailleurs ce que révèle le cas du mal: Dieu n'y concourt pas.

76 Ockham, Ordinatio II, q. 3-4 (v, 72-73): non potest demonstrari quod aliquis effectus producitur a causa secunda: quia licet semper ad approximationem ignis combustibili sequatur combustio, cum hoc tamen potest stare quod ignis non sit eius causa. Quia Deus potuit ordinasse quod semper ad praesentiam ignis passo approximato ipse solus causaret combustionem. [...] Per nulllum effectum potest probari quod aliquis sit homo, maxime per nullum effectum qui apparent in nobis, quia omnia quae videmus in homine potest angelus incorporatus facere, sicut comedere, bibere, etc. [...] Ideo non est mirabile si non possit demonstrari quod aliquid sit causa. Dico tunc quod [Deus] dicitur agere medantibus causis secundis quia coagit cum illis vel producit illud quod producunt causae secundae, et ita immediate sicut causae secundae. (Je souligne.) 
qu' il y ait une cause finie derrière les effets, une substance derrière les phénomènes, un homme derrière le visage d' homme.

Ce rapprochement a été étudié dans un article classique de W. Courtenay sur l'occasionalisme et le nominalisme ${ }^{77}$. J' ajouterai que, certes, de fait, Ockham est un nominaliste, mais rien ne dit qu' une théorie des universaux soit impliquée par cette doctrine de la causalité. Il n' est pas nécessaire d'être nominaliste pour avoir cette conception désarticulée de la causalité. La mutation semble s'esquisser déjà chez Duns Scot, qui affirme notamment que, par sa puissance absolue, «Dieu peut scier avec une scie molle ${ }^{78}$. Duns Scot formulait cet argument pour répondre à l' argument de Thomas: «si un couteau ne coupait pas, il aurait en vain son tranchant». Désormais, le tranchant du couteau n' est pas essentiel à son action - sous le régime de la puissance absolue, la forme des causes secondes n' est pas nécessairement la cause de leur action.

Ce nouveau modèle, comme l' a montré J. Schmutz, met en place une autonomie des causes secondes: il élimine la hiérarchie des causes secondes, illustrée par le premier modèle, où la cause première causait «à l'intime» (pour parler comme Augustin repris par Thomas) des causes secondes. Désormais, même si la cause première concourt aux actes positifs des causes secondes, à commencer par leur vouloir, elle ne le fait que par une influence générale, et sans déterminer son vouloir. C' est dans ce nouveau cadre que le libre arbitre est pensable. Dieu concourt à tous les actes humains, mais l'homme est abandonné à son ordre propre pour déterminer ses actions, même si c' est contre la volonté de Dieu ${ }^{79}$. Ce modèle des causes partielles concourantes s' est imposé dans les écoles jésuites, jusque dans l' œuvre de Molina, Dieu ne prêtant que son «concours général» aux causes finies, donc à toutes les décisions des libertés humaines ${ }^{80}$. Mais alors, c'est la liberté finie qui détermine le choix de ses actions: «le concours général de Dieu [...] dépend du libre arbitre humain», et non l'inverse ${ }^{81}$. Et ainsi, c' est l'hypothèse d'un ordre naturel du vouloir, cohérent et indépendant d' une causalité surnaturelle, qui gagne en cohérence et en solidité.

En définitive, l'articulation métaphysique de la cause première et de la cause seconde, telle qu'elle fut instituée par le Livre des causes, est essentielle à la pensée médiévale de la liberté, dans sa relation à Dieu.

77 Courtenay 1973, p. 77-94.

78 Duns Scot, Ordinatio IV, d. 1, §.319 (XI, 113).

79 Schmutz 200o, p. 241-243, cite en ce sens des textes de Duns Scot, Ockham et Gabriel Biel.

8o Molina, Concordia II, 26, 15 (éd. Rabeneck 1953, p. 170).

81 Molina, Concordia II, 29, 9 (éd. Rabeneck 1953, p. 189); cf. Schmutz 200o, p. 260. 
Pour cela, il a fallu d'abord écarter la dimension émanatiste que le Liber devait au néoplatonisme de Proclus, sur un point très précis: l'idée que le premier Principe transmettait son pouvoir créateur à la cause seconde, car cela revenait à maintenir la doctrine platonicienne des hypostases. Ainsi pouvait se déployer le premier modèle d'interprétation du Liber de causis, à partir des analyses de saint Thomas d'Aquin: la cause première cause à la fois l'être et l' agir des causes secondes. - Si on l' applique dans toute la rigueur de sa structure d'éminence et d'émanation, cette articulation permet de penser Dieu comme agissant au plus intime de la volonté humaine, cause seconde de l'accomplissement de sa providence. La contingence de l'action de la volonté n' est alors qu' un cas particulier de la contingence des causes secondes.

Mais contre Thomas, Siger met en question l'idée que la cause seconde n' ajoute rien à la cause première. Et pour sa part, à partir de sa propre lecture des deux concepts clés, Duns Scot propose une théorie de la causalité plus complexe et plus diversifiée, où le jeu des causes totales subordonnées est remplacé par celui des causes partielles concourantes. Le but de cette réforme de la théorie est d' abord de fonder la liberté de la volonté finie en nous obligeant à penser la cause première comme agissant de manière contingente. Mais il ouvre une sorte de béance: on ne voit plus très bien en quoi, lorsque la cause seconde est une volonté libre, la cause première contribue encore à son action.

Utilisant toujours le Liber de causis, Ockham éclaircit ce que Scot laissait dans l'ombre. Si Dieu n'est pas cause de mon vouloir, il reste qu' il collabore à mon action comme une cause partielle concourante. Mais s'il ne peut pas m'empêcher de vouloir ce que je veux, nous sommes des partenaires égaux. Dieu et l'homme sont causes partielles de l'action, mais Dieu et l'homme peuvent vouloir ou ne pas vouloir y contribuer, et aucun ne déclenche la volonté de l' autre. Nous sommes sur le même plan, si bien qu' on peut à la fois dire que nous agissons ensemble et que nous entrons en concurrence.

Ainsi, même chez les auteurs qui subvertissent la métaphysique néoplatonicienne de l'émanation, le Liber de causis continue, en subduction, à imposer ses concepts principaux: les concepts de cause première et de cause seconde. Il en résulte deux interprétations cohérentes et alternatives, qui débouchent conjointement sur deux représentations distinctes de la causalité et de la liberté82.

82 Une autre version de cet article a paru dans O. Boulnois, Généalogie de la liberté, Paris, Seuil, 2021, p. 398-419. 


\section{Bibliographie}

\section{Sources primaires}

Anselme, De potestate, Fragmenta philosophica II, in R.W. Southern, F.S. Schmitt, $M e-$ morials of Saint Anselm, London, British Academy, 1969.

Anselme, De potestate, trad. fr. A. Galonnier, in M. Corbin, L'œuvre de S. Anselme de Cantorbéry, t. 4, Paris, Cerf, 1990.

Aristote, De interpretatione, ed. H. Weidemann, Berlin, De Gruyter, 2014.

Augustin, Les Confessions, éd. et trad. fr. A. Solignac, E. Tréhorel, G. Bouissou, Bibliothèque Augustinienne, t. 13-14, Paris, Institut d'Études Augustiniennes, 1962 (nouvelle éd. augmentée, 1992).

Augustin, Réplique en quatre livres à deux lettres des pélagiens, in Premières polémiques contre Julien, éd. et trad. fr. F.-J. Thonnard, E. Bleuzen, A.-C. De Veer, Bibliothèque Augustinienne, t. 23, Paris, Institut d'Études Augustiniennes, 1974.

Bonaventure, De scientia Christi, in Opera Omnia S. Bonaventurae, t. v, Quaracchi, Collegium Sancti Bonaventurae, 1891-1902.

Duns Scot, Lectura in Librum Secundum Sententiarum. Distinctiones 7-44, éd. Commissio Scotistica, in Opera omnia, t. XIX, Città del Vaticano, Typis Polyglottis Vaticanis, 1993 .

Duns Scot, Ordinatio, éd. Commissio Scotistica in Opera omnia, t. I-XIV, Città del Vaticano, Typis Polyglottis Vaticanis, 1950-2013.

Duns Scot, Reportatio Parisiensis, in Opera omnia, t. 22, Paris, Vivès, 1891-1895.

Duns Scot, Sur la connaissance de Dieu et l'univocité de l'étant, trad. fr. O. Boulnois, Paris, Presses Universitaires de France, 1988.

Dominicus Gundissalinus, De processione mundi, éd. G. Bülow, Von dem Hervorgange der Welt, in Beiträge zur Geschichte der Philosophie im Mittelalter 24/3 (1925), p. 156 .

Immanuel Kant, Kritik der reinen Vernunft, trad. fr. A.J.-L. Delamarre, F. Marty in CEuvres philosophiques, Paris, Gallimard, 1980.

La condamnation parisienne de 1277, éd. et trad. fr. D. Piché, C. Lafleur, Paris, Vrin, 2000.

Liberde causis: A. Pattin, «Le Liber de causis. Édition établie à l' aide de 90 manuscrits », dans Tijdschrift voor Philosophie, 28(1966), p. 9o-203; trad. fr. O. Boulnois, P. Magnard, B. Pinchard, J.-L. Solère, in La demeure de l'être, Autour d'un anonyme: étude et traduction du Liber de causis, Paris, Vrin, 1990.

Molina, Liberi arbitrii cum gratiae donis, divina praescientia, providentia, praedestinatione et reprobatione concordia, éd. J. Rabeneck, Oña / Madrid, Collegium Maximum s.I., 1953 .

Guillaume d'Ockham, Quaestiones variae, éd. G.I. Etzkorn, F.E. Kelley, J.C. Wey, in Guillelmi de Ockham Opera Theologica, t. VIII, Saint Bonaventure, N.Y., The Franciscan Institute, 1981. 
Guillaume d'Ockham, Quodlibeta septem, éd. J.C. Wey, in Guillelmi de Ockham Opera Theologica, t. Ix, Saint Bonaventure, N.Y., The Franscican Institute, 1980.

Pascal, Écrits sur la grâce, in CEuvres complètes, t. II, éd. M. Le Guern, Paris, Gallimard, 2000.

Plotinus, Enneads, ed. P. Henry, H.-R. Schwyzer, Leiden, Brill, 1951-1973.

Proclus, Elements of Theology, éd. E.R. Dodds, Oxford, Clarendon Press, $1963^{2}$.

Proclus, Eléments de théologie, trad. fr. J. Trouillard, Paris, Aubier, 1965.

Roger Bacon, Quaestiones supra Librum de causis, in Opera Hactenus inedita Rogeri Baconi, t. XII, éd. R. Steele, Oxford, Clarendon Press, 1935.

Siger de Brabant, Quaestiones super Librum de causis, éd. A. Marlasca, Louvain / Paris, Publications universitaires / Béatrice-Nauwelaerts, 1972.

Siger de Brabant, Quaestiones in libros Meteorum, éd. J.-J. Duin in La doctrine de la providence dans les écrits de Siger de Brabant, Louvain, Éditions de l'Institut Superieur de Philosophie, 1954 .

Thomas d'Aquin, In Librum de causis expositio, éd. C. Pera, Turin, Marietti, 1955.

Thomas d' Aquin, Super Librum de causis expositio, éd. H.-D. Saffrey, Fribourg / Louvain, Société philosophique / E. Nauwelaerts, 1954 [Paris, Vrin, 2002²].

Thomas d'Aquin, Quaestiones disputatae de potentia Dei, éd. R.M. Spiazzi, Turin, Marietti, 1949.

Thomas d'Aquin, Scriptum super libros Sententiarum / super Sententiis Magistri Petri Lombardi, t. I-II, éd. P. Mandonnet, Paris, Lethielleux, 1929.

Thomas d'Aquin, Summa contra Gentiles, éd. Leon. t. 13-15, Rome, Typis Riccardi Garroni, 1918-1930.

Thomas d'Aquin, Summae theologiae, Prima Secundae. QQ. 1-LXX, éd. Leon., t. 6, Rome, Typographia poliglotta S.C. de Propaganda Fide, 1891.

Thomas d'Aquin, Summa theologiae. Prima Secundae, QQ. LXXI-CXIV, éd. Leon., t. 7, Rome, Typographia poliglotta S.C. de Propaganda Fide, 1897.

\section{Sources secondaires}

Calma, D. (2003), «Siger de Brabant et Thomas, note sur l'histoire d'un plagiat», dans Freiburger Zeitschrift für Philosophie und Theologie 5o (1/2), p. 118-135.

Calma, D. (2019), «Sine secundaria: Thomas d'Aquin, Siger de Brabant et les débats sur l' occasionalisme», dans D. Calma (éd.), Reading Proclus and the Book of Causes. Vol. 1. Western Scholarly Networks and Debates, Leyde, Brill, p. 268-30o.

Chantraine, P. (1968), Dictionnaire étymologique de la langue grecque, Paris, Klincksieck, 1968.

Counet, J.-M. (2019), «Duns Scot et le Liber de causis», dans D. Calma (éd.), Reading Proclus and the Book of Causes. Vol. ı. Western Scholarly Networks and Debates Leyde, Brill, p. 251-267.

Courtenay, W. (1973), «The Critique on Natural Causality in the Mutakallimun and Nominalism», dans Harvard Theological Review 66, p. 77-94. 
D'Ancona, C. (1995), Recherches sur le Livre des causes, Paris, Vrin.

de Franceschi, S. (2018), Thomisme et théologie moderne, L'École de saint Thomas à l'épreuve de la querelle de la grâce (XVII ${ }^{e}-X V I I I^{e}$ siècle), Paris, Perpignan.

Dhont, R.-C. (1947), Le problème de la préparation à la grâce. Débuts de l'école franciscaine, Paris, Éditions franciscains.

Gilson, E. (1926-1927), «Pourquoi S. Thomas a-t-il critiqué S. Augustin?», dans Archives d'Histoire Doctrinale et Littéraire du Moyen Age 1, p. 5-127.

Imbach, R. (1996), «Notule sur le commentaire du Liber de causis de Siger de Brabant et ses rapports avec Thomas d'Aquin », dans Freiburger Zeitschrift für Philosophie und Theologie 43, p. 304-323.

Imbach, R., Putallaz, F.-X., (1997), Profession: Philosophe. Siger de Brabant, Paris, Cerf.

Libera, A. de (2007), Archéologie du sujet, I. Naissance du sujet, Paris, Vrin.

Libera, A. de (1990), «Albert le Grand et Thomas d'Aquin interprètes du Liber de causis», dans Revue des sciences philosophiques et théologiques 74, p. 347-378.

Madec, G. (1987-1988), «Introduction à la doctrine augustinienne. Principes de spiritualité », dans École pratique des hautes études, Section des sciences religieuses, Annuaire 96, p. 308-309.

Muralt, A. de (1993), L'enjeu de la philosophie médiévale. Études thomistes, scotistes, occamiennes et grégoriennes, Leyde / New York / Cologne, Brill.

Perler, D., Rudolph, U. (200o), Occasionalismus. Theorien der Kausalität im arabischislamischen und europäische Denken, Göttingen, Vandenhoeck und Ruprecht.

Schmutz, J. (2000), «La doctrine médiévale des causes et la théologie de la nature pure (XIII ${ }^{\mathrm{e}}-\mathrm{XVII}^{\mathrm{e}}$ siècle)», in Revue thomiste 101, p. 217-264.

Vignaux, P. (1948), Nominalisme au XIV siècle, Montréal, Université de Montréal. Institut d'études médiévales. 